\title{
Association between dietary raw garlic intake and newly diagnosed nonalcoholic fatty liver disease: a population-based study
}

\author{
Shunming Zhang ${ }^{1, *}$, Yeqing Gu ${ }^{1, *}$, Liu Wang ${ }^{1}$, Qing Zhang ${ }^{2}$, Li Liu ${ }^{2}$, Min Lu ${ }^{3}$, Ge Meng ${ }^{1}$, Zhanxin Yao ${ }^{1,4}$, \\ Hongmei Wu' ${ }^{1}$, Yang Xia', Xue Bao', Honglei Wang', Hongbin Shi², Shaomei Sun², Xing Wang ${ }^{2}$, Ming Zhou'², \\ Qiyu Jia ${ }^{2}$, Kun Song ${ }^{2}$, Huiling Xiang ${ }^{3}$ and Kaijun Niu' ${ }^{1,2,5,6}$
}

\begin{abstract}
${ }^{1}$ Nutritional Epidemiology Institute and School of Public Health, Tianjin Medical University, Tianjin, China, ${ }^{2}$ Health Management Centre, Tianjin Medical University General Hospital, Tianjin, China, ${ }^{3}$ Department of Gastroenterology, Tianjin Third Central Hospital, Tianjin, China, ${ }^{4}$ Tianjin Institute of Health and Environmental Medicine, Tianjin, China, ${ }^{5}$ Tianjin Key Laboratory of Environment, Nutrition and Public Health, Tianjin, China, and ${ }^{6}$ Center for International Collaborative Research on Environment, Nutrition and Public Health, Tianjin, China
\end{abstract}

\begin{abstract}
Background and Aims: The protective effect of garlic against nonalcoholic fatty liver disease (NAFLD) has been reported in animal studies. However, in humans, the association between garlic consumption and NAFLD is unclear. The study sought to explore the association between habitual raw garlic intake and newly diagnosed NAFLD among Chinese adults.

Methods: We performed a study of 11,326 men and 12,780 women aged 20-90 years. Habitual food intake was assessed using a validated and standardized 100-item food frequency questionnaire. Diagnosis of NAFLD was based on the liver ultrasonography and self-reported alcohol intake. Multiple logistic regression was used to evaluate the association of raw garlic intake with newly diagnosed NAFLD.

Results: The prevalence of newly diagnosed NAFLD was $28.9 \%$ in men and $10.1 \%$ in women, respectively. In men, the fully adjusted odds ratios ( $95 \%$ confidence interval) of having NAFLD across increasing frequency of raw garlic intake were 1.00 (reference) for $<1$ time/week, $0.81(0.73,0.90)$ for $1-3$ times/week, $0.66(0.54,0.80)$ for $4-6$ times/week, and $0.71(0.55,0.90)$ for $\geq 7$ times/week ( $P$ for trend $<0.0001$ ). The odds ratio for NAFLD associated with each $1 \mathrm{~g}$ of raw garlic/1000 kcal was $0.93(0.90,0.97)$ in men. In women, no significant association between raw garlic intake and NAFLD was identified. These associations between raw garlic intake and NAFLD were consistent in several sensitivity analyses.

Conclusions: Frequent consumption of raw garlic is inversely associated with NAFLD in Chinese men. Further investigations are needed to confirm this finding.
\end{abstract}

\section{Introduction}

Nonalcoholic fatty liver disease (NAFLD) refers to a wide spectrum of liver disorders, ranging from steatosis to nonalcoholic steatohepatitis, fibrosis, and cirrhosis (1). NAFLD is the predominant cause of chronic liver diseases and it is strongly associated with cardiovascular disease
European Journal of Endocrinology (2019) 181, 591-602 
Dietary modifications are beneficial in the prevention of NAFLD. Garlic (Allium sativum) is widely consumed in many countries and cultures for centuries. It contains many organosulfur compounds such as S-allylcysteine, allicin, diallyl sulfide, diallyl disulfide and diallyl trisulfide (5). Animal studies have shown that garlic essential oil and its major organosulfur component (diallyl disulfide) could protect against NAFLD through by ameliorating lipid metabolic disorders and oxidative stress (6). Moreover, allicin is the principal sulfur-containing compounds obtained from raw garlic (5). Many studies have suggested that allicin has beneficial effects on oxidative damage $(7$, 8 ) and inflammation (9), both of which play an important role in the development of NAFLD $(10,11)$. Furthermore, human studies and animal models have shown that garlic has hypolipidemic effects $(12,13)$ and can improve insulin sensitivity (14), thereby playing a direct role in NAFLD prevention (11). Finally, intestinal microflora plays an important role in the pathogenesis of NAFLD (15, 16). According to a recent study, garlic polysaccharides can change the microbiota equilibrium (17). Therefore, we speculated that dietary raw garlic intake may have a beneficial effect on the prevention of NAFLD.

Although a randomized clinical trial (18) and several animal studies $(6,19,20,21,22,23)$ have suggested that garlic preparations may have a protective effect against NAFLD, potential effects of raw garlic intake on NAFLD have not been evaluated in population-based studies. On the other hand, the consumption of fresh and raw garlic in China is high, which provides an exceptional opportunity to investigate the association between raw garlic consumption and NAFLD. In the present study, therefore, we assessed the association between dietary raw garlic intake and newly diagnosed NAFLD in a large population of Chinese adults.

\section{Subjects and methods}

\section{Study participants}

We used data from the Tianjin Chronic Low-grade Systemic Inflammation and Health (TCLSIH) cohort study. The details of the TCLSIH study have been previously described (24). The protocol of this study was approved by the Institutional Review Board of the Tianjin Medical University and all participants gave their informed consent to participate in this study.

This cross-sectional study included 31,834 participants who had received health examinations during 2013-2016.
Subjects who were newly diagnosed with NAFLD were identified by the results of annual medical examinations and/or self-reported history of the disease. Participants diagnosed with alcoholic fatty liver disease and other liver diseases (including chronic hepatitis B or C, operations on the liver, autoimmune liver diseases, cirrhotic or liver cancer, etc.) were identified by the results of their annual medical examinations and/or self-reported history of diseases. We excluded those participants who were previously diagnosed with NAFLD based on the results of their annual medical examination $(n=4949)$ and participants with missing dietary data $(n=196)$. Moreover, those with a history of CVD $(n=1113)$ or cancer $(n=255)$ were excluded because of important dietary changes after the diagnosis (25). Those who had a history of other liver diseases $(n=134)$ or alcoholic fatty liver disease $(n=1081)$ were also excluded. After these exclusions, a total of 24,106 subjects (mean (standard deviation) age: 40.6 (11.9) years; men, $47.0 \%$ ) were included in the current analysis. The flowchart of study participants is given in Fig. 1.

\section{Definition of NAFLD}

All participants underwent real-time ultrasonography examination as part of the health examinations. Fatty liver was diagnosed by abdominal ultrasonography

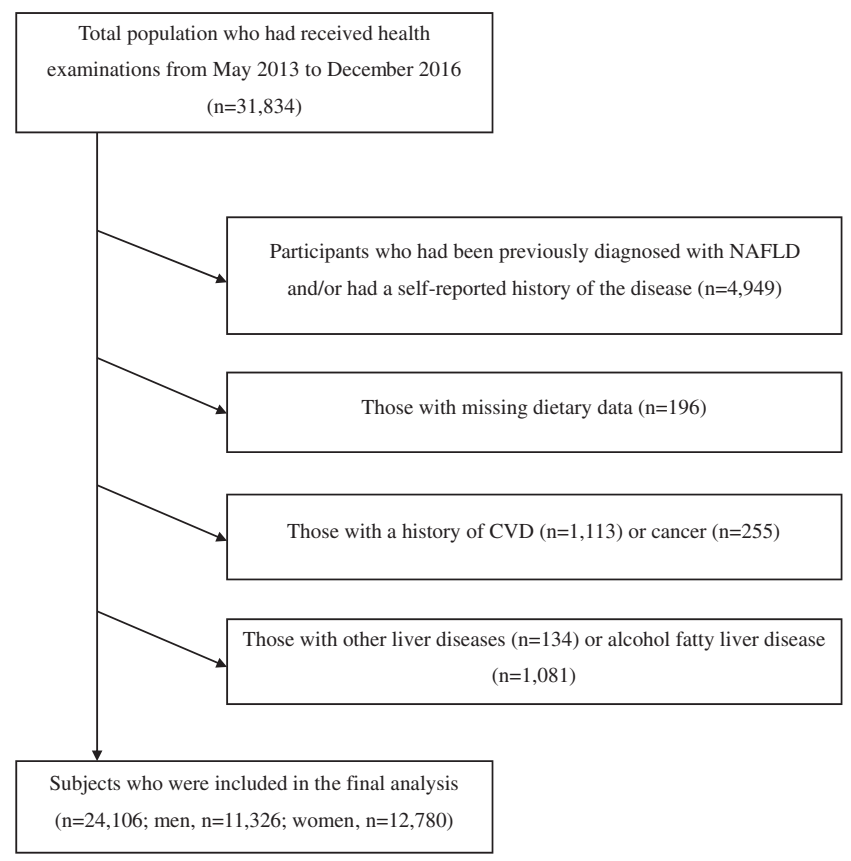

Figure 1

Selection of subjects for the study. CVD, cardiovascular disease; NAFLD, nonalcoholic fatty liver disease. 
using standardized criteria performed by experienced sonographers. Diagnosis of fatty liver disease required the presence of at least two of the following three abnormal findings (26): hyperechogenity of liver tissue ('bright liver') when compared to the hypoechogenity of the kidneys, vascular blurring, and deep attenuation of ultrasound signal. Participants with sonographic fatty liver disease and the weekly alcohol intake of $<140 \mathrm{~g}$ for men and $<70 \mathrm{~g}$ for women were defined as having NAFLD (27). Inter-observer variations for NAFLD status (yes or no) were evaluated in a subsample of 200 participants from the TCLSIH study. The Kappa coefficient was 0.90, and the total agreement was $96.4 \%$. Furthermore, as a confirmatory analysis to the ultrasound-established NAFLD, participants who had NAFLD and elevated serum alanine aminotransferase (ALT) levels (ALT $>30 \mathrm{U} / \mathrm{L}$ for men and $>19 \mathrm{U} / \mathrm{L}$ for women) were defined as NAFLD with elevated ALT $(28,29)$.

\section{Assessment of dietary intake}

Dietary intake was assessed using a valid and reliable food frequency questionnaire (FFQ), which included 100 items of foods with specified serving sizes. The FFQ assessed consumption frequency and portion sizes of various foods over the past month by checking one of seven frequency categories ranging from 'almost never' to ' $\geq 2$ times/day' for foods (including total onions, including both raw and cooked, as well as raw garlic) and eight frequency categories ranging from 'almost never' to ' $\geq 4$ times/ day' for beverages (including soft drinks). The FFQ was validated against 4-day weighed dietary records (WDRs) including 3 weekdays and 1 weekend day and the data from two FFQs collected approximately 3 months apart using a random sampling of 150 participants from the TCLSIH study (30). The participants were randomly selected from different subgroups (age: 20-30, 30-40, $40-50,50-60,60-70$, and $>70$ years), and at least ten men and ten women were included in each subgroup. The characteristics of the 150 participants were similar to that of the entire study population. Spearman correlation coefficients between the WDRs and the FFQ results were 0.49 for energy intake, $0.35-0.54$ for nutrients (n-3 fatty acid, fat, and carbohydrate), and 0.69 for raw garlic ( 0.70 for men and 0.68 for women). Spearman's rank correlation coefficients between two FFQs were 0.68 for energy intake, 0.62-0.79 for food items (fruits, vegetables, sweet foods, and beverages), and 0.78 for raw garlic. The mean daily intakes of energy and nutrients were calculated using an ad hoc computer program for the FFQ, which was based on the 2009 Chinese Food Composition Table. In the present study, to assess the potential confounding effect of overall diet quality on the association between raw garlic intake and NAFLD, three major dietary patterns (sweet pattern, healthy pattern, and animal food pattern) were derived by factor analysis after removing the raw garlic and total onions (31). Some unhealthy foods such as red meat or processed meat, preserved egg, and soft drinks were included in the animal food pattern.

Participants reported their frequency of raw garlic and total onions consumption over the previous month by selecting the following options: almost never, $<1$ time/week, 1 time/week, 2-3 times/week, 4-6 times/week, 1 time/day, and $\geq 2$ times/day. Based on the frequency distribution of responses, the categories of raw garlic consumption were divided into four groups: <1 time/week, 1-3 times/week, 4-6 times/week, and $\geq 7$ times/week.

\section{Assessment of other variables}

The anthropometric indices (height, weight, and waist circumference (WC)) were measured by well-trained investigators using a standard protocol. Body mass index (BMI) was calculated as the body weight (kg) divided by the square of the body height $(\mathrm{m})$. The sociodemographic characteristics, including age, sex, household income, educational level, and occupation, were self-reported. Smoking status, drinking status, individual and family history of disease (including CVD, hypertension, hyperlipidemia, and diabetes) were also assessed by a questionnaire survey.

The blood samples were drawn $12 \mathrm{~mL}$ from fasting subjects. Serum total cholesterol (TC) and triglycerides (TGs) were measured by enzymatic methods, low-density lipoprotein cholesterol (LDL) was measured by the polyvinyl sulfuric acid precipitation method, and highdensity lipoprotein cholesterol (HDL) was measured by the chemical precipitation method. Fasting blood glucose (FBG) was measured using the glucose oxidase method. Serum ALT was measured by the International Federation of Clinical Chemistry method. These measurements were performed by using reagents from Roche diagnostics on an automatic biochemistry analyzer (Roche Cobas 8000 modular analyzer).

According to the criteria of the JNC 7, hypertension was defined as systolic blood pressure (SBP) of $\geq 140 \mathrm{mmHg}$ and/or diastolic blood pressure (DBP) of $\geq 90 \mathrm{mmHg}$ or having a history of hypertension. PA was assessed using the short form of the International Physical Activity 
Questionnaire (32). PA was estimated as metabolic equivalents in hours per week (MET-hour/week).

\section{Statistical analysis}

All analyses were stratified by sex because the interaction between sex and raw garlic intake (continuous; g/day per $1000 \mathrm{kcal}(33))$ in the multivariate model was statistically significant ( $P$ for the interaction was $<0.0001$ ). Analysis of covariance for continuous variables and logistic regression analysis for categorical variables were used to compare participant characteristics among different categories of raw garlic consumption or status of NAFLD. The characteristics of the participants were presented as geometric mean (95\% confidence interval, CI) for continuous variables, or as percentage for categorical variables. Multiple logistic regression models were used to assess the association between raw garlic intake and NAFLD (or NAFLD with elevated ALT). Raw garlic intake was assessed as both categorical and continuous variables; in the categorical analyses, the odds ratio (OR) and corresponding 95\% CI were calculated using the $<1$ time/week group as the reference, whereas in the continuous analyses, the OR (95\% CI) was calculated as $1 \mathrm{~g} /$ day per $1000 \mathrm{kcal}$. In model 1, we adjusted for age and BMI. In model 2, we additionally adjusted for educational level, occupation, household income, PA, and total energy intake. In a final model, we further adjusted for all variables in model 2 in addition to three major dietary patterns (raw garlic and total onions were not included in the calculation) and total onion intake. We constructed a directed acyclic graph (DAG) to justify the inclusion of these confounders (which were associated with both exposures and the outcomes in this study) (34). Figure 2 shows the DAG was derived from previous literature $(35,36)$ and expert knowledge. To identify the minimally sufficient adjustment set, we used the program, DAGitty (37).

We tested the interactions of raw garlic intake (as a continuous variable (33)) with all covariates by the inclusion of cross-product terms in the fully adjusted model. Moreover, variance inflation factors (VIFs) were used to detect multicollinearity among covariates in the final model. VIFs exceeding 10 were a sign of multicollinearity. To minimize the potential reverse causation, we did several sensitivity analyses: (1) excluded 11,274 participants who had hypertension, hyperlipidemia, and/or diabetes from the analyses; (2) excluded 4,915 participants who have changed their lifestyles in the past 5 years, with changes in dietary intake, drinking status, smoking status, PA, and sleeping; (3) excluded 1,775 participants with long- term medication use. Furthermore, we used the E-value methodology of VanderWeele and Ding to quantify residual confounding $(38,39)$.

The $P$ values for linear trends were calculated by using the categories of raw garlic consumption $(<1$ time/week: 1; 1-3 times/week: 2; 4-6 times/week: 3; and $\geq 7$ times/ week: 4) as an ordinal variable. Bonferroni-corrected $P$ values were used in multiple tests. SAS version 9.4 (SAS Institute, Inc.) was used for all statistical analyses. Twosided $P<0.05$ was considered statistically significant.

\section{Results}

\section{Study participant characteristics}

In total, 11,326 men (age: $41.0 \pm 12.2$ years) and 12,780 women (age: $40.3 \pm 11.6$ years) participated in this study. Table 1 shows the age-adjusted participant characteristics according to their categories of raw garlic consumption. In both sexes, participants with higher raw garlic intake were more likely to be older, had higher BMI, consumed total energy and onions, and more adherence to three major dietary patterns (sweet pattern, healthy pattern, and animal food pattern). In men, those with higher raw garlic intake tended to be physically active, were more likely to smoke, had a higher proportion of everyday drinkers, but had a lower proportion of ex drinkers. In addition, the proportion of non-drinkers had U-shaped association across raw garlic intake categories in men. In women, the proportion of sometime drinkers had reverse U-shaped association, whereas the proportion of nondrinkers had U-shaped association across raw garlic intake categories.

Table 2 describes participants' characteristics in relation to NAFLD. Compared with men and women without NAFLD, those with NAFLD had higher age, BMI, WC, TC, TG, LDL, SBP, DBP, FBG, ALT, and lower HDL and total energy intake. However, there were also some differences between men and women. Men with NAFLD had lower sweet pattern scores, animal food pattern scores, PA, and a lower proportion of everyday drinkers, but had a higher proportion of current smokers, nonsmokers, ex-drinkers, and non-drinkers; they were less likely to be employed as managers, had lower education level. Women with NAFLD had a higher proportion of ex-drinkers and a lower proportion of everyday drinkers, sometime drinkers, were more likely to be employed not as managers or professionals and had lower education level and household income. 


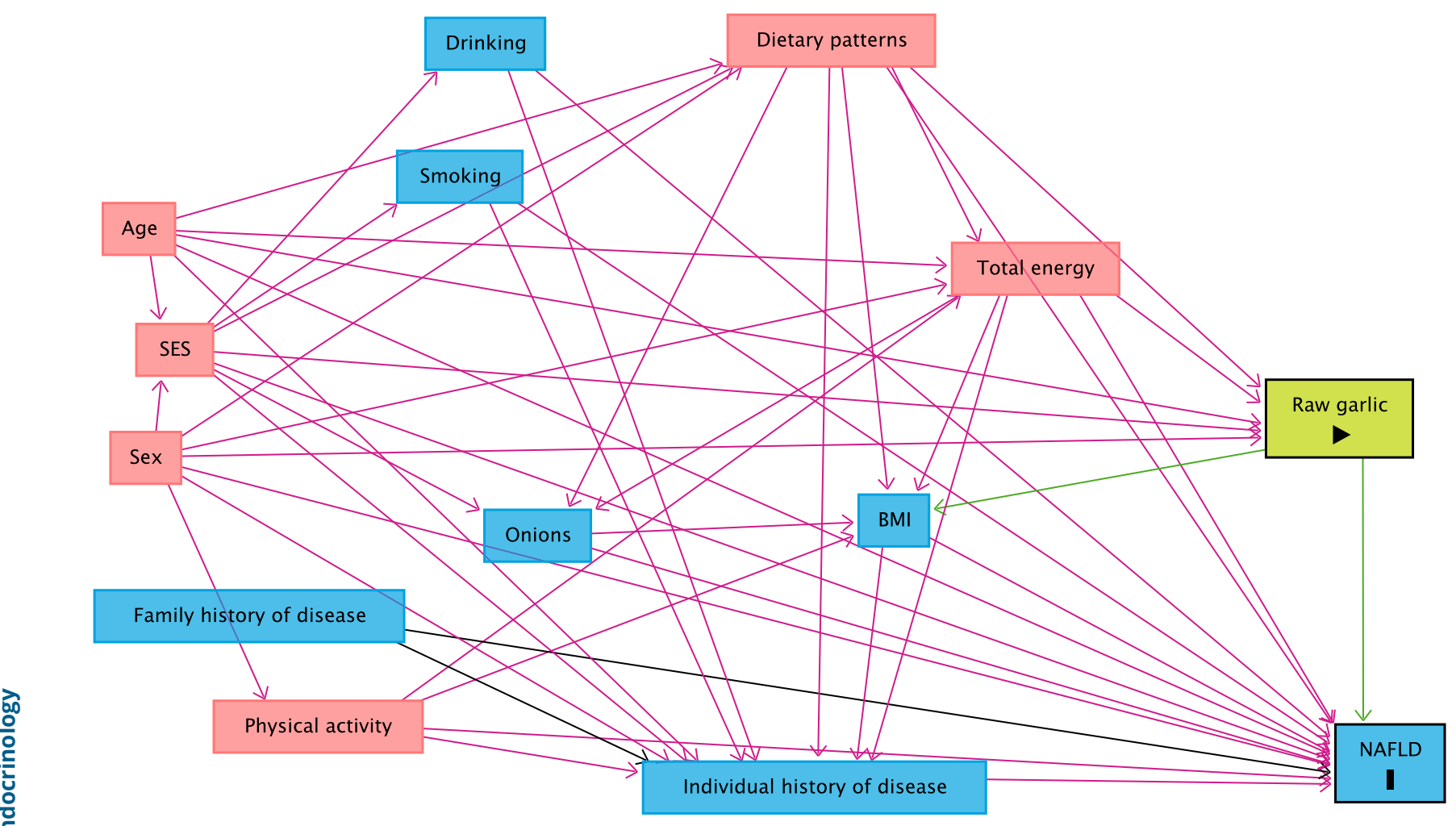

\section{Figure 2}

Directed acyclic graph (DAG) derived from previous literature and expert knowledge. Arrows represent causal associations. Raw garlic is exposure, and NAFLD is the outcome. BMI, body mass index; NAFLD, nonalcoholic fatty liver disease; SES, socioeconomic status (including educational level, occupation, and household income).

\section{Association between raw garlic intake and NAFLD}

Table 3 presents the associations between raw garlic consumption categories and newly diagnosed NAFLD. Adjusting for age and BMI, the ORs (95\% CIs) for NAFLD across categories of raw garlic consumption were 1.00 (reference) for $<1$ time/week, $0.78(0.71,0.87)$ for $1-3$ times/week, $0.62(0.52,0.75)$ for $4-6$ times/week, and $0.62(0.50,0.78)$ for $\geq 7$ times/week ( $P$ for trend $<0.0001)$ in men. However, the association was not statistically significant in women $(P$ for trend $=0.14)$. After additional adjustment for sociodemographic status, lifestyle factors, total energy intake, and dietary intake, these associations were not altered. The fully adjusted ORs (95\% CIs) for NAFLD across categories of raw garlic consumption were 1.00 (reference) for $<1$ time/week, $0.81(0.73,0.90)$ for 1-3 times/week, $0.66(0.54,0.80)$ for $4-6$ times/week, and $0.71(0.55,0.90)$ for $\geq 7$ times/week ( $P$ for trend $<0.0001)$ in men; the corresponding ORs (95\% CIs) were 1.00 (reference), $1.12(0.96,1.30), 1.17(0.88,1.55)$, and 1.17 $(0.86,1.58)$ ( $P$ for trend $=0.12)$ in women. An increase of $1 \mathrm{~g} / 1000 \mathrm{kcal}$ per day of raw garlic was inversely associated with NAFLD in men (OR=0.93; 95\% CI: 0.90, 0.97), but no in women $(\mathrm{OR}=1.05 ; 95 \% \mathrm{CI}$ : 0.98, 1.11). In both sexes, we did not observe any significant interactions between raw garlic intake and covariates in the final model. For multicollinearity, all VIF values ranged from 1.01 to 1.56. Furthermore, in sensitivity analyses to minimize the potential reverse causation, similar results were also observed (Supplementary Tables 1, 2 and 3, see section on supplementary data given at the end of this article). The sensitivity analysis using E-value methodology to assess the potential effect of unmeasured confounding showed that the E-value for an OR of 0.71 (95\% CI: 0.55, 0.90 ) was 1.66 for point estimate and 1.29 for the upper confidence limit.

\section{Association between raw garlic intake and NAFLD with elevated ALT}

As a confirmatory analysis to the ultrasound-established NAFLD, similar associations were observed in NAFLD with elevated ALT (Table 4). The fully adjusted ORs (95\% CIs) 
Table 1 Age-adjusted participant characteristics according to categories of raw garlic consumption $(n=24,106)^{*}$.

\begin{tabular}{|c|c|c|c|c|c|}
\hline \multirow[b]{2}{*}{ Characteristics } & \multicolumn{4}{|c|}{ Categories of raw garlic consumption (Men, $n=11,326$ ) } & \multirow[b]{2}{*}{$P$ for trend } \\
\hline & $<1$ time/week & $1-3$ times/week & 4-6 times/week & $\geq 7$ times/week & \\
\hline No. of subjects & 4653 & 5165 & 918 & 590 & - \\
\hline Age (years) & $37.9(37.6,38.2)$ & $39.8(39.4,40.1)$ & $41.0(40.2,41.7)$ & $45.3(44.3,46.4)$ & $<0.0001$ \\
\hline BMI $\left(\mathrm{kg} / \mathrm{m}^{2}\right)$ & $25.0(24.9,25.1)$ & $25.2(25.2,25.3)$ & $25.3(25.1,25.5)$ & $25.4(25.1,25.7)$ & 0.02 \\
\hline $\mathrm{WC}(\mathrm{cm})$ & $87.7(87.5,88.0)$ & $88.3(88.1,88.6)$ & $88.3(87.8,88.9)$ & $88.5(87.8,89.2)$ & 0.06 \\
\hline $\mathrm{TC}(\mathrm{mmol} / \mathrm{L})$ & $4.65(4.62,4.67)$ & $4.67(4.64,4.69)$ & $4.64(4.59,4.70)$ & $4.63(4.57,4.70)$ & 0.94 \\
\hline TG (mmol/L) & $1.27(1.25,1.29)$ & $1.29(1.27,1.31)$ & $1.30(1.25,1.35)$ & $1.30(1.25,1.36)$ & 0.17 \\
\hline LDL (mmol/L) & $2.74(2.72,2.76)$ & $2.74(2.72,2.76)$ & $2.70(2.65,2.75)$ & $2.73(2.67,2.80)$ & 0.20 \\
\hline HDL (mmol/L) & $1.20(1.19,1.21)$ & $1.21(1.20,1.21)$ & $1.20(1.18,1.22)$ & $1.18(1.15,1.20)$ & 0.72 \\
\hline $\mathrm{SBP}(\mathrm{mmHg})$ & $122.7(122.2,123.1)$ & $123.0(122.6,123.4)$ & $123.0(122.0,123.9)$ & $123.7(122.5,124.8)$ & 0.56 \\
\hline $\mathrm{DBP}(\mathrm{mmHg})$ & $77.5(77.2,77.8)$ & $78.1(77.8,78.4)$ & $78.2(77.5,78.9)$ & $78.4(77.6,79.3)$ & 0.051 \\
\hline FBG (mmol/L) & $5.16(5.14,5.19)$ & $5.18(5.16,5.20)$ & $5.18(5.14,5.23)$ & $5.25(5.19,5.32)$ & 0.44 \\
\hline $\operatorname{ALT}(U / L)$ & $21.6(21.2,21.9)$ & $21.5(21.2,21.9)$ & $21.5(20.8,22.3)$ & $22.2(21.2,23.2)$ & 0.93 \\
\hline Physical activity (MET-h/week) & $9.91(9.51,10.3)$ & $11.2(10.7,11.6)$ & $13.3(12.1,14.6)$ & $11.7(10.1,12.8)$ & $<0.0001$ \\
\hline Total energy intake (kcal/day) & $1985.4(1970.3,2000.6)$ & $2096.7(2081.5,2111.9)$ & $2183.8(2146.7,2221.7)$ & $2310.7(2261.5,2361.1)$ & $<0.0001$ \\
\hline 'Sweets' dietary pattern score & $-0.11(-0.13,-0.08)$ & $0.06(0.04,0.09)$ & $0.49(0.43,0.55)$ & $1.26(1.18,1.34)$ & $<0.0001$ \\
\hline $\begin{array}{l}\text { 'Vegetables' dietary } \\
\text { pattern score }\end{array}$ & $-0.21(-0.24,-0.18)$ & $-0.05(-0.08,-0.02)$ & $0.13(0.07,0.20)$ & $0.24(0.16,0.32)$ & $<0.0001$ \\
\hline $\begin{array}{l}\text { 'Animal foods' dietary } \\
\text { pattern score }\end{array}$ & $0.10(0.07,0.13)$ & $0.29(0.26,0.32)$ & $0.37(0.30,0.44)$ & $0.42(0.33,0.50)$ & $<0.0001$ \\
\hline Total onions intake (g/day) & $3.17(3.07,3.28)$ & $5.23(5.08,5.38)$ & $7.05(6.61,7.52)$ & $7.57(6.98,8.20)$ & $<0.0001$ \\
\hline \multicolumn{6}{|l|}{ Smoking status (\%) } \\
\hline Current smoker & 33.4 & 37.2 & 37.9 & 40.0 & $<0.01$ \\
\hline Ex-smoker & 8.05 & 8.85 & 9.98 & 14.0 & 0.051 \\
\hline Non-smoker & 58.6 & 54.0 & 52.1 & 46.0 & $<0.0001$ \\
\hline \multicolumn{6}{|l|}{ Drinking status (\%) } \\
\hline Everyday drinker & 4.64 & 7.26 & 9.64 & 10.7 & $<0.0001$ \\
\hline Sometime drinker & 69.6 & 73.8 & 72.6 & 68.3 & 0.10 \\
\hline Ex-drinker & 12.6 & 9.52 & 9.31 & 7.98 & $<0.0001$ \\
\hline Non-drinker & 13.1 & 9.40 & 8.43 & 13.1 & $<0.0001$ \\
\hline $\begin{array}{l}\text { Education level } \\
\qquad(\geq \text { College graduate, \%) }\end{array}$ & 69.4 & 69.1 & 63.2 & 56.5 & 0.45 \\
\hline \multicolumn{6}{|l|}{ Occupation (\%) } \\
\hline Managers & 41.2 & 43.9 & 42.5 & 41.1 & 0.22 \\
\hline Professionals & 21.1 & 21.4 & 19.5 & 20.8 & 0.43 \\
\hline Other & 37.7 & 34.7 & 38.0 & 38.2 & 0.06 \\
\hline $\begin{array}{l}\text { Household income } \\
\qquad(\geq 10,000 \text { Yuan, \%) }\end{array}$ & 35.2 & 35.8 & 34.9 & 34.7 & 0.57 \\
\hline
\end{tabular}

for NAFLD with elevated ALT across the categories of raw garlic consumption were 1.00 (reference) for $<1$ time/ week, $0.89(0.78,1.02)$ for $1-3$ times/week, $0.74(0.57$, $0.94)$ for $4-6$ times/week, and $0.71(0.51,0.98)$ for $\geq 7$ times/week ( $P$ for trend $<0.01$ ) in men; the fully adjusted ORs (95\% CIs) were 1.00 (reference), 1.11 (0.90, 1.37), $1.34(0.92,1.90)$, and $1.16(0.79,1.69)(P$ for trend $=0.16)$.

\section{Discussion}

The results of this study showed that dietary raw garlic intake was significantly associated with a lower prevalence of NAFLD among Chinese men, but not women. To our knowledge, this was the first study to assess the association between raw garlic consumption and NAFLD in such a large general population.

In this study, we adjusted for confounding factors identified using the DAG programbased on prior knowledge, which can avoid collider bias and overadjustment bias (34, 35). The minimal sufficient adjustment sets for estimating the effect of raw garlic intake on NAFLD included the following confounders: age, sex, BMI, PA, educational level, occupation, household income, total energy intake, dietary patterns, and onions. First, we made an adjustment for age and BMI. Second, we made further adjustments 


\begin{tabular}{|c|c|c|c|c|}
\hline \multicolumn{4}{|c|}{ Categories of raw garlic consumption (Women, $n=12,780$ ) } & \multirow[b]{2}{*}{$P$ for trend ${ }^{\dagger}$} \\
\hline$<1$ time/week & 1-3 times/week & 4-6 times/week & $\geq 7$ times/week & \\
\hline 7146 & 4272 & 745 & 617 & - \\
\hline $37.3(37.0,37.5)$ & $40.0(39.7,40.3)$ & $40.8(40.0,41.6)$ & $44.8(43.9,45.8)$ & $<0.0001$ \\
\hline $22.7(22.6,22.8)$ & $23.0(22.9,23.1)$ & $23.0(22.8,23.2)$ & $23.1(22.8,23.3)$ & 0.02 \\
\hline $76.6(76.4,76.7)$ & $77.2(76.9,77.4)$ & $77.1(76.5,77.6)$ & $77.1(76.5,77.8)$ & 0.10 \\
\hline $4.61(4.59,4.63)$ & $4.63(4.60,4.65)$ & $4.58(4.52,4.63)$ & $4.59(4.53,4.65)$ & 0.22 \\
\hline $0.90(0.89,0.91)$ & $0.91(0.90,0.92)$ & $0.89(0.86,0.92)$ & $0.91(0.88,0.94)$ & 0.49 \\
\hline $2.56(2.54,2.57)$ & $2.59(2.57,2.61)$ & $2.54(2.49,2.59)$ & $2.56(2.51,2.62)$ & 0.49 \\
\hline $1.54(1.53,1.54)$ & $1.52(1.51,1.53)$ & $1.51(1.49,1.54)$ & $1.51(1.48,1.54)$ & 0.14 \\
\hline $114.4(114.1,114.7)$ & $114.8(114.4,115.2)$ & $114.9(113.9,115.9)$ & $114.9(113.9,116.0)$ & 0.32 \\
\hline $71.3(71.0,71.5)$ & $71.6(71.3,71.8)$ & $71.7(71.0,72.4)$ & $71.8(71.0,72.5)$ & 0.24 \\
\hline $4.95(4.93,4.96)$ & $4.97(4.95,4.98)$ & $4.97(4.93,5.01)$ & $4.95(4.90,4.99)$ & 0.30 \\
\hline $12.9(12.7,13.0)$ & $13.1(12.9,13.3)$ & $13.1(12.6,13.5)$ & $13.4(12.9,14.0)$ & 0.41 \\
\hline $7.59(7.33,7.86)$ & $7.96(7.61,8.32)$ & $8.02(7.21,8.92)$ & $8.47(7.53,9.50)$ & 0.33 \\
\hline $1846.2(1833.3,1859.2)$ & $1972.6(1954.9,1990.5)$ & $2094.2(2049.4,2139.9)$ & $2246.9(2193.9,2301.2)$ & $<0.0001$ \\
\hline$-0.22(-0.24,-0.19)$ & $-0.08(-0.11,-0.05)$ & $0.30(0.23,0.37)$ & $1.02(0.94,1.09)$ & $<0.0001$ \\
\hline$-0.04(-0.07,-0.02)$ & $0.13(0.10,0.16)$ & $0.34(0.27,0.41)$ & $0.72(0.64,0.79)$ & $<0.0001$ \\
\hline$-0.29(-0.31,-0.27)$ & $-0.11(-0.13,-0.08)$ & $-0.03(-0.09,0.04)$ & $0.02(-0.05,0.09)$ & $<0.0001$ \\
\hline $2.61(2.54,2.69)$ & $4.50(4.35,4.65)$ & $6.02(5.58,6.49)$ & $7.58(6.99,8.21)$ & $<0.0001$ \\
\hline 1.36 & 1.66 & 1.46 & 2.53 & 0.30 \\
\hline 0.75 & 0.65 & 0.73 & 0.72 & 0.60 \\
\hline 97.9 & 97.7 & 97.8 & 96.8 & 0.57 \\
\hline 0.59 & 0.87 & 0.68 & 0.99 & 0.46 \\
\hline 38.0 & 42.2 & 44.2 & 38.7 & 0.001 \\
\hline 10.2 & 8.52 & 10.3 & 11.1 & 0.30 \\
\hline 51.2 & 48.4 & 44.8 & 49.3 & $<0.0001$ \\
\hline 67.2 & 62.6 & 62.7 & 47.4 & 0.19 \\
\hline 41.3 & 41.3 & 40.1 & 36.6 & 0.74 \\
\hline 13.1 & 12.5 & 14.0 & 11.1 & 0.84 \\
\hline 45.6 & 46.2 & 45.9 & 52.3 & 0.87 \\
\hline 34.7 & 33.9 & 33.0 & 30.5 & 0.38 \\
\hline
\end{tabular}

*Values are geometric mean (95\% confidence interval) or percentage unless otherwise indicated. ALT, alanine aminotransferase; BMI, body mass index; DBP, diastolic blood pressure; FBG, fasting blood glucose; HDL, high-density lipoprotein cholesterol; LDL, low-density lipoprotein cholesterol; MET, metabolic equivalent; SBP, systolic blood pressure; TC, total cholesterol; TG, triglycerides; WC, waist circumference.

${ }^{\dagger}$ Analysis of covariance or logistic regression analysis. $P$ for trend was calculated using the categories of raw garlic consumption ordinal as the predictor variable.

for educational level, occupation, household income, $\mathrm{PA}$, and total energy intake. However, adjustments for these confounding factors did not change the observed associations. Third, to test for the potential influence of nutritional quality of diets on the association between raw garlic intake and NAFLD, we additionally adjusted for three major dietary patterns (removing the raw garlic and total onions). In addition, because onions have a similar composition to garlic (40), we also adjusted for total intake of onions. However, the adjustment for these dietary factors did not change the associations between raw garlic consumption and NAFLD. Furthermore, animal studies have shown that garlic+onion have a better and significant effect on NAFLD (21). However, the interaction between raw garlic intake and total onions intake for NAFLD was not statistically significant $(P=0.95$ for men and $P=0.75$ for women). This could be due to the fact that people in Tianjin, China hardly eat raw onions, whereas cooking decreases the main bioactive compound, sulfur compounds, contained in onions (41). Finally, our results remained robust in several sensitivity analyses.

Many previous studies that investigated the association between garlic intake and NAFLD were performed in animal models $(6,19,20,21,22,23)$, and therefore, unable to determine whether garlic intake has also an effect on NAFLD in humans. Only one clinical trial showed that 
Table 2 Age-adjusted participant characteristics by NAFLD status $(n=24,106)^{*}$.

\begin{tabular}{|c|c|c|c|c|c|c|}
\hline \multirow[b]{2}{*}{ Characteristics } & \multicolumn{2}{|c|}{ NAFLD status (men, $n=11,326$ ) } & \multirow[b]{2}{*}{ P value $^{\dagger}$} & \multicolumn{2}{|c|}{ NAFLD status (women, $n=12,780$ ) } & \multirow[b]{2}{*}{ P value $^{\dagger}$} \\
\hline & No & Yes & & No & Yes & \\
\hline No. of subjects & 8048 & 3278 & - & 11,485 & 1295 & - \\
\hline Age (years) & $39.0(38.8,39.2)$ & $40.3(39.9,40.7)$ & $<0.0001$ & $37.9(37.7,38.1)$ & $46.8(46.1,47.5)$ & $<0.0001$ \\
\hline $\mathrm{BMI}\left(\mathrm{kg} / \mathrm{m}^{2}\right)$ & $24.1(24.1,24.2)$ & $27.9(27.8,28.0)$ & $<0.0001$ & $22.4(22.4,22.5)$ & $26.8(26.6,27.0)$ & $<0.0001$ \\
\hline$W C(\mathrm{~cm})$ & $85.5(85.3,85.7)$ & $94.7(94.4,95.0)$ & $<0.0001$ & $75.9(75.7,76.0)$ & $85.7(85.2,86.2)$ & $<0.0001$ \\
\hline $\mathrm{TC}(\mathrm{mmol} / \mathrm{L})$ & $4.57(4.55,4.59)$ & $4.86(4.83,4.89)$ & $<0.0001$ & $4.59(4.58,4.61)$ & $4.81(4.76,4.85)$ & $<0.0001$ \\
\hline TG (mmol/L) & $1.12(1.11,1.13)$ & $1.76(1.73,1.79)$ & $<0.0001$ & $0.86(0.85,0.87)$ & $1.37(1.34,1.40)$ & $<0.0001$ \\
\hline LDL (mmol/L) & $2.67(2.66,2.69)$ & $2.88(2.86,2.91)$ & $<0.0001$ & $2.54(2.53,2.55)$ & $2.80(2.76,2.84)$ & $<0.0001$ \\
\hline $\mathrm{HDL}(\mathrm{mmol} / \mathrm{L})$ & $1.27(1.26,1.28)$ & $1.06(1.05,1.06)$ & $<0.0001$ & $1.57(1.56,1.57)$ & $1.25(1.24,1.27)$ & $<0.0001$ \\
\hline $\mathrm{SBP}(\mathrm{mmHg})$ & $120.8(120.5,121.1)$ & $128.2(127.6,128.7)$ & $<0.0001$ & $113.5(113.2,113.7)$ & $124.7(123.9,125.5)$ & $<0.0001$ \\
\hline $\mathrm{DBP}(\mathrm{mmHg})$ & $76.3(76.0,76.5)$ & $81.9(81.6,82.3)$ & $<0.0001$ & $70.8(70.6,70.9)$ & $77.3(76.7,77.9)$ & $<0.0001$ \\
\hline FBG (mmol/L) & $5.09(5.07,5.10)$ & $5.41(5.38,5.44)$ & $<0.0001$ & $4.91(4.90,4.92)$ & $5.39(5.35,5.42)$ & $<0.0001$ \\
\hline $\operatorname{ALT}(\mathrm{U} / \mathrm{L})$ & $18.7(18.5,18.9)$ & $30.6(30.1,31.2)$ & $<0.0001$ & $12.5(12.4,12.6)$ & $18.5(18.1,19.0)$ & $<0.0001$ \\
\hline $\begin{array}{l}\text { Physical activity } \\
\text { (MET-h/week) }\end{array}$ & $12.0(11.6,12.3)$ & $11.3(10.8,11.8)$ & 0.02 & $8.75(8.54,8.96)$ & $9.05(8.41,9.73)$ & 0.40 \\
\hline $\begin{array}{l}\text { Total energy intake } \\
\text { (kcal/day) }\end{array}$ & $2080.8(2068.6,2093.0)$ & $2035.0(2016.4,2053.8)$ & $<0.0001$ & $1924.1(1913.4,1934.9)$ & $1879.8(1848.0,1912.1)$ & 0.01 \\
\hline $\begin{array}{l}\text { 'Sweets' dietary pattern } \\
\text { score }\end{array}$ & $0.03(0.00,0.05)$ & $-0.06(-0.10,-0.03)$ & $<0.0001$ & $0.00(-0.02,0.02)$ & $-0.01(-0.07,0.05)$ & 0.71 \\
\hline $\begin{array}{l}\text { 'Vegetables' dietary } \\
\text { pattern score }\end{array}$ & $-0.01(-0.03,0.02)$ & $0.01(-0.02,0.05)$ & 0.39 & $0.00(-0.01,0.02)$ & $-0.04(-0.1,0.01)$ & 0.11 \\
\hline $\begin{array}{l}\text { 'Animal foods' dietary } \\
\text { pattern score }\end{array}$ & $0.01(-0.01,0.04)$ & $-0.03(-0.07,0.00)$ & 0.02 & $0.00(-0.01,0.02)$ & $-0.04(-0.09,0.02)$ & 0.17 \\
\hline $\begin{array}{l}\text { Total onions intake } \\
\text { (g/day) }\end{array}$ & $4.53(4.43,4.65)$ & $4.37(4.20,4.54)$ & 0.11 & $3.52(3.44,3.60)$ & $3.41(3.18,3.64)$ & 0.38 \\
\hline \multicolumn{7}{|l|}{ Smoking status (\%) } \\
\hline Current smoker & 34.9 & 38.2 & $<0.01$ & 8.85 & 8.96 & 0.57 \\
\hline Ex-smoker & 56.3 & 52.9 & 0.98 & 1.45 & 2.23 & 0.27 \\
\hline Non-smoker & 0.68 & 1.07 & 0.01 & 97.9 & 96.7 & 0.28 \\
\hline \multicolumn{7}{|l|}{ Drinking status (\%) } \\
\hline Everyday drinker & 8.10 & 2.76 & $<0.0001$ & 0.75 & 0.40 & 0.03 \\
\hline Sometime drinker & 71.4 & 72.4 & 0.28 & 40.3 & 35.8 & $<0.01$ \\
\hline Ex-drinker & 10.0 & 12.4 & $<0.001$ & 9.61 & 10.2 & $<0.01$ \\
\hline Non-drinker & 10.5 & 12.4 & $<0.01$ & 49.4 & 53.6 & 0.12 \\
\hline $\begin{array}{l}\text { Education level } \\
\qquad \begin{array}{l}\text { ( } \geq \text { College } \\
\text { graduate, } \% \text { ) }\end{array}\end{array}$ & 69.6 & 64.3 & $<0.0001$ & 67.0 & 41.3 & $<0.0001$ \\
\hline \multicolumn{7}{|l|}{ Occupation (\%) } \\
\hline Managers & 43.6 & 39.9 & $<0.001$ & 41.9 & 33.6 & $<0.01$ \\
\hline Professionals & 21.5 & 20.2 & 0.25 & 13.2 & 9.60 & $<0.01$ \\
\hline Other & 34.9 & 39.9 & $<0.0001$ & 45.0 & 56.8 & $<0.0001$ \\
\hline $\begin{array}{l}\text { Household income } \\
\qquad(\geq 10,000 \text { Yuan, \%) }\end{array}$ & 35.2 & 35.9 & 0.37 & 34.8 & 28.0 & $<0.01$ \\
\hline
\end{tabular}

*Values are geometric mean (95\% confidence interval) or percentage unless otherwise indicated.

ALT, alanine aminotransferase; BMI, body mass index; DBP, diastolic blood pressure; FBG, fasting blood glucose; HDL, high-density lipoprotein cholesterol; LDL, low-density lipoprotein cholesterol; MET, metabolic equivalent; NAFLD, nonalcoholic fatty liver disease; SBP, systolic blood pressure; TC, total cholesterol; TG, triglycerides; WC, waist circumference.

${ }^{\dagger}$ Analysis of covariance or logistic regression analysis.

15 weeks garlic powder supplementation could reduce body weight and fat mass among NAFLD subjects, whose consisted of 98 volunteers (including men and women) aged 20-70 years (18). In addition, a 12-week doubleblind, randomized, placebo-controlled trial in 75 adults, aged 20-75 years, found that fermented garlic extract may be beneficial in improving for hepatic dysfunction (42). Our findings, obtained in men, were consistent with prior results suggesting that garlic intake has a profoundly beneficial effect on NAFLD. Therefore, this study indicated that raw garlic intake could be part of a preventive strategy to combat NAFLD in men. However, previous studies also showed that high dose of garlic extract could elicit prooxidant conditions or induce morphological changes in the liver and kidneys $(43,44)$. Notably, these extracts, which is not the same as actual garlic, tend to be highly concentrated. Whether a high raw garlic intake would have a harmful effect on human health is unclear. Therefore, further population studies examining a safe dose range for raw garlic intake are warranted.

In China, Chinese meals and side dishes often include fresh and raw garlic, which is generally chopped 
Table 3 Associations between raw garlic consumption and NAFLD $(n=24,106)^{*}$.

\begin{tabular}{l}
\hline Logistic regression models \\
\hline Men $(n=11,326)$ \\
Total number of participants \\
NAFLD, $n(\%)$ \\
Model $1^{\ddagger}$ \\
Model $2^{\|}$ \\
Model $3^{\text {" }}$ \\
Women $(n=12,780)$ \\
Total number of participants \\
NAFLD, $n(\%)$ \\
Model $1^{\ddagger}$ \\
Model $2^{\|}$ \\
Model $3^{\text {m }}$
\end{tabular}

\begin{tabular}{|c|c|c|c|}
\hline \multicolumn{4}{|c|}{ Categories of raw garlic consumption } \\
\hline$<1$ time/week & 1-3 times/week & 4-6 times/week & $\geq 7$ times/week \\
\hline 4653 & 5165 & 918 & 590 \\
\hline $1424(30.6)$ & $1458(28.2)$ & $237(25.8)$ & $159(26.9)$ \\
\hline 1.00 (reference) & $0.78(0.71,0.87)^{\S}$ & $0.62(0.52,0.75)$ & $0.62(0.50,0.78)$ \\
\hline 1.00 (reference) & $0.79(0.71,0.88)$ & $0.62(0.51,0.75)$ & $0.63(0.50,0.79)$ \\
\hline 1.00 (reference) & $0.81(0.73,0.90)$ & $0.66(0.54,0.80)$ & $0.71(0.55,0.90)$ \\
\hline 7,146 & 4,272 & 745 & 617 \\
\hline $613(8.58)$ & $496(11.6)$ & 89 (11.9) & $97(15.7)$ \\
\hline 1.00 (reference) & $1.14(0.98,1.32)^{\S}$ & $1.11(0.83,1.46)$ & $1.16(0.87,1.53)$ \\
\hline 1.00 (reference) & $1.12(0.96,1.30)$ & $1.15(0.86,1.52)$ & $1.14(0.85,1.51)$ \\
\hline 1.00 (reference) & $1.12(0.96,1.30)$ & $1.17(0.88,1.55)$ & $1.17(0.86,1.58)$ \\
\hline
\end{tabular}

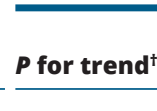

\section{$1 \mathrm{~g} / 1000 \mathrm{kcal}$} increase

\begin{tabular}{c} 
increase \\
\hline 11,326 \\
$3278(28.9)$ \\
$0.93(0.89,0.97)$ \\
$0.92(0.88,0.95)$ \\
$0.93(0.90,0.97)$
\end{tabular}

*NAFLD, nonalcoholic fatty liver disease.

${ }^{\dagger}$ Obtained by using multiple logistic regression analysis. The $P$ values for trend were calculated by using the categories of raw garlic consumption $(<1$ time/week: 1; 1-3 times/week: 2; 4-6 times/week: 3; and $\geq 7$ times/week: 4) as an ordinal variable.

${ }^{\ddagger}$ Adjusted for age (continuous; years) and BMI (continuous; $\mathrm{kg} / \mathrm{m}^{2}$ ).

${ }^{\S}$ Odds ratio (95\% confidence interval) (all such values).

"Additionally adjusted for educational level (categorical: < or $\geq$ college graduate), occupation (categorical; managers, professionals, and other), household income (categorical: $\leq$ or > 10,000 Yuan), physical activity (continuous; MET-hour/week), and total energy intake (continuous; kcal/day).

"Further adjusted for total onions intake (continuous; g/day per $1000 \mathrm{kcal}$ ) and three major dietary pattern scores (raw garlic and total onions were not included in the calculation).

or crushed. Additionally, some Chinese people chew raw fresh garlic cloves when they are eating noodles. Because chopping, crushing or chewing raw garlic releases more allicin than by preparing and consuming cooked garlic (45), individuals can ingest a high amount of allicin from dietary raw garlic intake. Accumulated evidence suggested that allicin has the strong antioxidant activity and antiinflammatory effects $(7,8,46)$, which have preventive effects against NAFLD. On the other hand, raw garlic intake can decrease insulin resistance (14), which is a key pathogenic factor of NAFLD (47). Furthermore, an important cause of NAFLD is dyslipidemia (47).

Table 4 Associations between raw garlic consumption and NAFLD with elevated ALT $(n=24,106)^{*}$.

\begin{tabular}{|c|c|c|c|c|c|c|}
\hline \multirow[b]{2}{*}{ Logistic regression models } & \multicolumn{4}{|c|}{ Categories of raw garlic consumption } & \multirow[b]{2}{*}{$P$ for trend ${ }^{\dagger}$} & \multirow{2}{*}{$\begin{array}{c}1 \mathrm{~g} / 1000 \mathrm{kcal} \\
\text { increase }\end{array}$} \\
\hline & $<1$ time/week & 1-3 times/week & 4-6 times/week & $\geq 7$ times/week & & \\
\hline \multicolumn{7}{|l|}{$\operatorname{Men}(n=11,326)$} \\
\hline Total number of participants & 4653 & 5165 & 918 & 590 & - & 11,326 \\
\hline NAFLD with elevated ALT, $n(\%)$ & $682(14.7)$ & $698(13.5)$ & 109 (11.9) & $62(10.5)$ & - & $1551(13.7)$ \\
\hline Model $1^{\ddagger}$ & 1.00 (reference) & $0.90(0.79,1.02)^{\S}$ & $0.73(0.57,0.93)$ & $0.67(0.49,0.90)$ & $<0.001$ & $0.93(0.89,0.98)$ \\
\hline Model 2\| & 1.00 (reference) & $0.88(0.78,1.00)$ & $0.70(0.54,0.88)$ & $0.66(0.48,0.89)$ & $<0.001$ & $0.94(0.89,0.98)$ \\
\hline Model 39 & 1.00 (reference) & $0.89(0.78,1.02)$ & $0.74(0.57,0.94)$ & $0.71(0.51,0.98)$ & $<0.01$ & $0.95(0.90,0.99)$ \\
\hline \multicolumn{7}{|l|}{ Women $(n=12,780)$} \\
\hline Total number of participants & 7146 & 4272 & 745 & 617 & - & 12,780 \\
\hline NAFLD with elevated ALT, $n(\%)$ & $278(3.89)$ & $224(5.24)$ & $45(6.04)$ & $46(7.46)$ & - & $593(4.64)$ \\
\hline Model $1^{\ddagger}$ & 1.00 (reference) & $1.14(0.94,1.40)^{\S}$ & $1.29(0.89,1.83)$ & $1.25(0.86,1.78)$ & 0.08 & $1.07(0.99,1.15)$ \\
\hline Model 2\| & 1.00 (reference) & $1.13(0.92,1.38)$ & $1.34(0.94,1.89)$ & $1.20(0.83,1.70)$ & 0.10 & $1.07(1.00,1.14)$ \\
\hline Model 3" & 1.00 (reference) & $1.11(0.90,1.37)$ & $1.34(0.92,1.90)$ & $1.16(0.79,1.69)$ & 0.16 & $1.06(0.98,1.14)$ \\
\hline 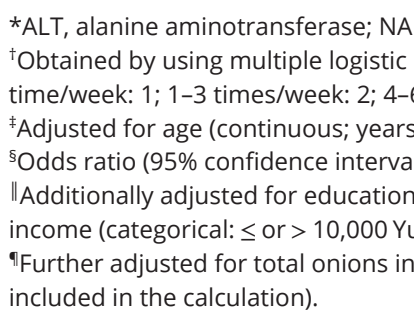 & $\begin{array}{l}6 \text { times/week: 3; and } \\
\text { s) and BMI (continuou } \\
\text { al) (all such values). } \\
\text { hal level (categorical: } \\
\text { uan), physical activity } \\
\text { ttake (continuous; g/d }\end{array}$ & $\begin{array}{l}\text { The } P \text { values for trend } \\
\geq 7 \text { times/week: } 4 \text { ) as } \\
\mathrm{us} \text {; } \mathrm{kg} / \mathrm{m}^{2} \text { ). } \\
<\text { or } \geq \text { college graduat } \\
\text { ( continuous; MET-hol } \\
\text { day per } 1000 \mathrm{kcal} \text { ) and }\end{array}$ & $\begin{array}{l}\text { e), occupation (categc } \\
\text { ar/week), and total en } \\
\text { three major dietary }\end{array}$ & $\begin{array}{l}\text { orical; managers, pro } \\
\text { nergy intake (continu } \\
\text { pattern scores (raw g }\end{array}$ & $\begin{array}{l}\text { fessionals, } \\
\text { ous; kcal/da } \\
\text { sarlic and to }\end{array}$ & $\begin{array}{l}\text { other), household } \\
\text { nions were not }\end{array}$ \\
\hline
\end{tabular}


Many animal studies have demonstrated that garlic extracts can lower serum lipids, lipoprotein profiles, and cholesterol by controlling biosynthesis of hepatic fatty acid and cholesterol $(48,49)$. Therefore, the inverse association between raw garlic consumption and NAFLD is biologically reasonable.

Interestingly, no association was found between raw garlic intake and NAFLD in women. A possible explanation for the sex differences is that estrogen signaling pathway might be mediators for NAFLD in women. Evidence has shown that estrogens have a protective effect against the development of NAFLD in women (50). However, animal studies found that sulfides in garlic inhibit estrogen receptor $\alpha$ (51). Meanwhile, studies demonstrated that estrogen receptor $\alpha$ is the primary mediator for estrogen signaling to protect against fatty liver (52). Therefore, the possible explanation for the lack of association between raw garlic intake and NAFLD may be that sulfides in garlic diminish the responsiveness of hormone-receptive tissue by inhibiting estrogen receptor $\alpha$. Further study is needed to determine the molecular mechanisms responsible for the lack of association between raw garlic consumption and NAFLD in women.

The major strengths of this study include adjustments for confounding factors identified by the DAG, large sample size, detailed measurements of lifestyles, and use of a validated FFQ for assessment of dietary intake. Moreover, we only included newly diagnosed NAFLD, which further reduced the potential effects of reverse causation in the present study. Meanwhile, this study has several limitations worth considering. First, this was a cross-sectional study, restricting causal inference. However, we performed several sensitivity analyses to minimize the potential reverse causation; results from these sensitivity analyses support our findings. More importantly, the inverse association between raw garlic intake and NAFLD is biologically reasonable, which strengthens the biological plausibility of our findings. Nevertheless, we admit that further longitudinal studies and trials are needed to confirm our findings. Second, although we adjusted for many confounding factors underlying causal assumptions, residual confounding factors could persist. However, the sensitivity analysis using E-value methodology showed that the observed OR of 0.71 could be explained away by an unmeasured confounder that was associated with both raw garlic intake and NAFLD by an OR of 1.66-fold each, above and beyond the measured confounders, but weaker confounding could not do so; the CI could be moved to include the null by an unmeasured confounder that was associated with both raw garlic intake and NAFLD by an OR of 1.29-fold each, above and beyond the measured confounders, but again, weaker confounding could not do so. Since an OR of 1.66 was much greater than any observed confounders in our study, it is unlikely that an unmeasured confounder would be able to overcome the effects of raw garlic intake on NAFLD in the current study. Nevertheless, the drawbacks of a cross-sectional study cannot be remedied by statistical methods. Thus, future prospective studies are warranted. Third, owing to the observational nature of this study, imprecision in the measurement of the included confounding factors cannot be excluded. Finally, we were unable to collect data on intake of cooked garlic or garlic products. Therefore, further studies are still required to determine the association between intake of cooked garlic or garlic products and NAFLD.

\section{Conclusion}

In conclusion, the higher intake of raw garlic was associated with a decreased prevalence of NAFLD in men, but not women. These results suggest that raw garlic intake may have a protective effect on NAFLD. Further prospective studies or clinical trials are needed to confirm the causality of the associations.

\section{Supplementary data}

This is linked to the online version of the paper at https://doi.org/10.1530/ EJE-19-0179.

\section{Declaration of interest}

The authors declare that there is no conflict of interest that could be perceived as prejudicing the impartiality of the research reported.

\section{Funding}

This study was supported by grants from the National Natural Science Foundation of China (No. 81673166).

\section{Author contribution statement}

S Z and $Y G$ analyzed data and wrote the paper. $L W, Q Z, L L, M L, G M, Z Y$, $\mathrm{H} \mathrm{W}, \mathrm{YX}, \mathrm{X} \mathrm{B}, \mathrm{H} \mathrm{W}, \mathrm{HS}, \mathrm{S} \mathrm{S}, \mathrm{XW}, \mathrm{M} \mathrm{Z}, \mathrm{Q} J, \mathrm{~K} \mathrm{~S}$, and $\mathrm{HX}$ conducted research. $\mathrm{K} \mathrm{N}$ designed research and had primary responsibility for final content. All authors read and approved the final manuscript.

\section{Acknowledgments}

The authors gratefully acknowledge all the people that have made this study. 


\section{References}

1 Angulo P. Nonalcoholic fatty liver disease. The New England Journal of Medicine 2002346 1221-1231. (https://doi.org/10.1056/ NEJMra011775)

2 Francque SM, van der Graaff D \& Kwanten WJ. Non-alcoholic fatty liver disease and cardiovascular risk: pathophysiological mechanisms and implications. Journal of Hepatology 201665 425-443. (https://doi. org/10.1016/j.jhep.2016.04.005)

3 Loomba R \& Sanyal AJ. The global NAFLD epidemic. Nature Reviews. Gastroenterology \& Hepatology 201310 686-690. (https://doi. org/10.1038/nrgastro.2013.171)

4 Abd El-Kader SM \& El-Den Ashmawy EM. Non-alcoholic fatty liver disease: the diagnosis and management. World Journal of Hepatology 20157 846-858. (https://doi.org/10.4254/wjh.v7.i6.846)

5 Bayan L, Koulivand PH \& Gorji A. Garlic: a review of potential therapeutic effects. Avicenna Journal of Phytomedicine 20144 1-14.

6 Lai YS, Chen WC, Ho CT, Lu KH, Lin SH, Tseng HC, Lin SY \& Sheen LY. Garlic essential oil protects against obesity-triggered nonalcoholic fatty liver disease through modulation of lipid metabolism and oxidative stress. Journal of Agricultural and Food Chemistry 201462 5897-5906. (https://doi.org/10.1021/jf500803c)

7 Chan JY, Yuen AC, Chan RY \& Chan SW. A review of the cardiovascular benefits and antioxidant properties of allicin. Phytotherapy Research: PTR 201327 637-646. (https://doi. org/10.1002/ptr.4796)

8 Prasad K, Laxdal VA, Yu M \& Raney BL. Antioxidant activity of allicin, an active principle in garlic. Molecular and Cellular Biochemistry 1995148 183-189. (https://doi.org/10.1007/ bf00928155)

9 Quintero-Fabian S, Ortuno-Sahagun D, Vazquez-Carrera M \& Lopez-Roa RI. Alliin, a garlic (Allium sativum) compound, prevents LPS-induced inflammation in 3T3-L1 adipocytes. Mediators of Inflammation 20132013 381815. (https://doi. org/10.1155/2013/381815)

10 Polimeni L, Del Ben M, Baratta F, Perri L, Albanese F, Pastori D, Violi F \& Angelico F. Oxidative stress: new insights on the association of non-alcoholic fatty liver disease and atherosclerosis. World Journal of Hepatology 20157 1325-1336. (https://doi.org/10.4254/wjh. v7.i10.1325)

11 Jung UJ \& Choi MS. Obesity and its metabolic complications: the role of adipokines and the relationship between obesity, inflammation, insulin resistance, dyslipidemia and nonalcoholic fatty liver disease. International Journal of Molecular Sciences 201415 6184-6223. (https://doi.org/10.3390/ijms15046184)

12 Ragavan G, Muralidaran Y, Sridharan B, Nachiappa Ganesh R \& Viswanathan P. Evaluation of garlic oil in nano-emulsified form: optimization and its efficacy in high-fat diet induced dyslipidemia in Wistar rats. Food and Chemical Toxicology : an International Journal Published for the British Industrial Biological Research Association 2017 105 203-213. (https://doi.org/10.1016/j.fct.2017.04.019)

13 Ashraf R, Aamir K, Shaikh AR \& Ahmed T. Effects of garlic on dyslipidemia in patients with type 2 diabetes mellitus. Journal of Ayub Medical College, Abbottabad: JAMC 200517 60-64.

14 Padiya R, Khatua TN, Bagul PK, Kuncha M \& Banerjee SK. Garlic improves insulin sensitivity and associated metabolic syndromes in fructose fed rats. Nutrition \& Metabolism 20118 53. (https://doi. org/10.1186/1743-7075-8-53)

15 Ma J, Zhou Q \& Li H. Gut microbiota and nonalcoholic fatty liver disease: insights on mechanisms and therapy. Nutrients 20179 E1124. (https://doi.org/10.3390/nu9101124)

16 Xue L, He J, Gao N, Lu X, Li M, Wu X, Liu Z, Jin Y, Liu J, Xu J, et al. Probiotics may delay the progression of nonalcoholic fatty liver disease by restoring the gut microbiota structure and improving intestinal endotoxemia. Scientific Reports 20177 45176. (https://doi. org/10.1038/srep45176)
17 Wang Y, Guan M, Zhao X \& Li X. Effects of garlic polysaccharide on alcoholic liver fibrosis and intestinal microflora in mice. Pharmaceutical Biology 201856 325-332. (https://doi.org/10.1080/13 880209.2018.1479868)

18 Soleimani D, Paknahad Z, Askari G, Iraj B \& Feizi A. Effect of garlic powder consumption on body composition in patients with nonalcoholic fatty liver disease: a randomized, double-blind, placebo-controlled trial. Advanced Biomedical Research 201652. (https://doi.org/10.4103/2277-9175.174962)

19 Qamar A, Usmani A, Waqar H, Siddiqui A \& Kumar H. Ameliorating effect of Allium sativum on high-fat diet induced fatty liver in albino rats. Pakistan Journal of Medical Sciences 201632 403-407. (https:// doi.org/10.12669/pjms.322.9025)

20 Qamar A, Siddiqui A \& Kumar H. Fresh garlic amelioration of highfat-diet induced fatty liver in albino rats. JPMA. The Journal of the Pakistan Medical Association 201565 1102-1107.

21 El-Din SH, Sabra AN, Hammam OA, Ebeid FA \& El-Lakkany NM. Pharmacological and antioxidant actions of garlic and.or onion in non-alcoholic fatty liver disease (NAFLD) in rats. Journal of the Egyptian Society of Parasitology 201444 295-308. (https://doi. org/10.12816/0006468)

22 Xiao J, Guo R, Fung ML, Liong EC, Chang RC, Ching YP \& Tipoe GL. Garlic-derived S-allylmercaptocysteine ameliorates nonalcoholic fatty liver disease in a rat model through inhibition of apoptosis and enhancing autophagy. Evidence-Based Complementary and Alternative Medicine: eCAM 20132013 642920. (https://doi. org $/ 10.1155 / 2013 / 642920$ )

23 Xiao J, Ching YP, Liong EC, Nanji AA, Fung ML \& Tipoe GL. Garlicderived S-allylmercaptocysteine is a hepato-protective agent in nonalcoholic fatty liver disease in vivo animal model. European Journal of Nutrition 201352 179-191. (https://doi.org/10.1007/s00394-0120301-0)

24 Zhang S, Fu J, Zhang Q, Liu L, Meng G, Yao Z, Wu H, Bao X, Gu Y, $\mathrm{Lu} \mathrm{M}$, et al. Association between nut consumption and nonalcoholic fatty liver disease in adults. Liver International: Official Journal of the International Association for the Study of the Liver 201939 1732-1741. (https://doi.org/10.1111/liv.14164)

25 Hu FB, Stampfer MJ, Rimm E, Ascherio A, Rosner BA, Spiegelman D $\&$ Willett WC. Dietary fat and coronary heart disease: a comparison of approaches for adjusting for total energy intake and modeling repeated dietary measurements. American Journal of Epidemiology 1999149 531-540. (https://doi.org/10.1093/oxfordjournals.aje. a009849)

26 Farrell GC, Chitturi S, Lau GK, Sollano JD \& Asia-Pacific Working Party on NAFLD. Guidelines for the assessment and management of non-alcoholic fatty liver disease in the Asia-Pacific region: executive summary. Journal of Gastroenterology and Hepatology 200722 775-777. (https://doi.org/10.1111/j.1440-1746.2007.05002.x)

27 Zeng MD, Fan JG, Lu LG, Li YM, Chen CW, Wang BY, Mao YM \& Chinese National Consensus Workshop on Nonalcoholic Fatty Liver Disease. Guidelines for the diagnosis and treatment of nonalcoholic fatty liver diseases. Journal of Digestive Diseases 20089 108-112. (https://doi.org/10.1111/j.1751-2980.2008.00331.x)

28 Ruhl CE \& Everhart JE. Elevated serum alanine aminotransferase and gamma-glutamyltransferase and mortality in the United States population. Gastroenterology 2009136 477-485 e411. (https://doi. org/10.1053/j.gastro.2008.10.052)

29 Prati D, Taioli E, Zanella A, Della Torre E, Butelli S, Del Vecchio E, Vianello L, Zanuso F, Mozzi F, Milani S, et al. Updated definitions of healthy ranges for serum alanine aminotransferase levels. Annals of Internal Medicine 2002137 1-10. (https://doi.org/10.7326/0003-4819137-1-200207020-00006)

30 Gu Y, Zhang S, Wang J, Chi VTQ, Zhang Q, Liu L, Meng G, Yao Z, $\mathrm{Wu} \mathrm{H}, \mathrm{Bao} \mathrm{X}$, et al. Relationship between consumption of raw garlic and handgrip strength in a large-scale adult population. Clinical Nutrition 2019 Epub. (https://doi.org/10.1016/j.clnu.2019.05.015) 
31 Xia Y, Xiang Q, Gu Y, Jia S, Zhang Q, Liu L, Meng G, Wu H, Bao X, Yu B, et al. A dietary pattern rich in animal organ, seafood and processed meat products is associated with newly diagnosed hyperuricaemia in Chinese adults: a propensity score-matched casecontrol study. The British Journal of Nutrition 2018119 1177-1184. (https://doi.org/10.1017/S0007114518000867)

32 Craig CL, Marshall AL, Sjostrom M, Bauman AE, Booth ML, Ainsworth BE, Pratt M, Ekelund U, Yngve A, Sallis JF, et al. International physical activity questionnaire: 12 -country reliability and validity. Medicine and Science in Sports and Exercise 200335 1381-1395. (https://doi.org/10.1249/01.MSS.0000078924.61453.FB)

33 Thoresen M. Spurious interaction as a result of categorization. BMC Medical Research Methodology 201919 28. (https://doi.org/10.1186/ s12874-019-0667-2)

34 Shrier I \& Platt RW. Reducing bias through directed acyclic graphs. BMC Medical Research Methodology 20088 70. (https://doi. org/10.1186/1471-2288-8-70)

35 Rohrig N, Strobl R, Muller M, Perz S, Kaab S, Martens E, Peters A, Linkohr B \& Grill E. Directed acyclic graphs helped to identify confounding in the association of disability and electrocardiographic findings: results from the KORA-Age study. Journal of Clinical Epidemiology 201467 199-206. (https://doi.org/10.1016/j. jclinepi.2013.08.012)

36 Alferink LJ, Kiefte-de Jong JC, Erler NS, Veldt BJ, Schoufour JD, de Knegt RJ, Ikram MA, Metselaar HJ, Janssen H, Franco OH, et al. Association of dietary macronutrient composition and non-alcoholic fatty liver disease in an ageing population: the Rotterdam Study. Gut 201968 1088-1098. (https://doi.org/10.1136/gutjnl-2017-315940)

37 Textor J, Hardt J \& Knuppel S. DAGitty: a graphical tool for analyzing causal diagrams. Epidemiology 201122 745. (https://doi.org/10.1097/ EDE.0b013e318225c2be)

38 VanderWeele TJ \& Ding P. Sensitivity analysis in observational research: introducing the E-value. Annals of Internal Medicine 2017 167 268-274. (https://doi.org/10.7326/M16-2607)

39 Haneuse S, VanderWeele TJ \& Arterburn D. Using the E-value to assess the potential effect of unmeasured confounding in observational studies. JAMA 2019321 602-603. (https://doi. org/10.1001/jama.2018.21554)

40 Galeone C, Pelucchi C, Levi F, Negri E, Franceschi S, Talamini R, Giacosa A \& La Vecchia C. Onion and garlic use and human cancer. The American Journal of Clinical Nutrition 200684 1027-1032. (https://doi.org/10.1093/ajcn/84.5.1027)

41 Nicastro HL, Ross SA \& Milner JA. Garlic and onions: their cancer prevention properties. Cancer Prevention Research 20158 181-189. (https://doi.org/10.1158/1940-6207.CAPR-14-0172)

42 Kim HN, Kang SG, Roh YK, Choi MK \& Song SW. Efficacy and safety of fermented garlic extract on hepatic function in adults with elevated serum gamma-glutamyl transpeptidase levels: a doubleblind, randomized, placebo-controlled trial. European Journal of
Nutrition 201756 1993-2002. (https://doi.org/10.1007/s00394-0161318-6)

43 Suru SM \& Ugwu CE. Comparative assessment of onion and garlic extracts on endogenous hepatic and renal antioxidant status in rat. Journal of Basic and Clinical Physiology and Pharmacology 201526 347-354. (https://doi.org/10.1515/jbcpp-2014-0088)

44 Banerjee SK, Maulik M, Manchanda SC, Dinda AK, Das TK \& Maulik SK. Garlic-induced alteration in rat liver and kidney morphology and associated changes in endogenous antioxidant status. Food and Chemical Toxicology : an International Journal Published for the British Industrial Biological Research Association 200139 793-797. (https://doi.org/10.1016/s0278-6915(01)00018-7)

45 Hosseini A \& Hosseinzadeh H. A review on the effects of Allium sativum (garlic) in metabolic syndrome. Journal of Endocrinological Investigation 201538 1147-1157. (https://doi.org/10.1007/s40618015-0313-8)

46 Li C, Lun WJ, Zhao XM, Lei S, Guo YD, Ma JY \& Zhi FC. Allicin alleviates inflammation of trinitrobenzenesulfonic acid-induced rats and suppresses P38 and JNK pathways in Caco- 2 cells. Mediators of Inflammation 20152015 434692. (https://doi. org/10.1155/2015/434692)

47 Gaggini M, Morelli M, Buzzigoli E, DeFronzo RA, Bugianesi E \& Gastaldelli A. Non-alcoholic fatty liver disease (NAFLD) and its connection with insulin resistance, dyslipidemia, atherosclerosis and coronary heart disease. Nutrients 20135 1544-1560. (https://doi. org/10.3390/nu5051544)

48 Ha AW, Ying T \& Kim WK. The effects of black garlic (Allium satvium) extracts on lipid metabolism in rats fed a high fat diet. Nutrition Research and Practice 20159 30-36. (https://doi.org/10.4162/ nrp.2015.9.1.30)

49 Mohammadi A \& Oshaghi EA. Effect of garlic on lipid profile and expression of LXR alpha in intestine and liver of hypercholesterolemic mice. Journal of Diabetes and Metabolic Disorders 201413 20. (https://doi.org/10.1186/2251-6581-13-20)

50 Ballestri S, Nascimbeni F, Baldelli E, Marrazzo A, Romagnoli D \& Lonardo A. NAFLD as a sexual dimorphic disease: role of gender and reproductive status in the development and progression of nonalcoholic fatty liver disease and inherent cardiovascular risk. Advances in Therapy 201734 1291-1326. (https://doi.org/10.1007/ s12325-017-0556-1)

51 Hahm ER \& Singh SV. Diallyl trisulfide inhibits estrogen receptoralpha activity in human breast cancer cells. Breast Cancer Research and Treatment 2014144 47-57. (https://doi.org/10.1007/s10549-0142841-x)

52 Hart-Unger S, Arao Y, Hamilton KJ, Lierz SL, Malarkey DE, Hewitt SC, Freemark M \& Korach KS. Hormone signaling and fatty liver in females: analysis of estrogen receptor alpha mutant mice. International Journal of Obesity 201741 945-954. (https://doi. org/10.1038/ijo.2017.50)

Received 10 March 2019

Revised version received 27 August 2019

Accepted 26 September 2019 